\title{
Comparison Between Mode Theory and Ray Theory of VLF Propagation
}

\author{
H. Volland
}

(November 10, 1960)

\begin{abstract}
It is shown that the field strength according to mode theory and ray theory in the VLF band are derivable from the same expression of the original vector potential, and the result of one theory is the analytic continuation of the other one in another range of convergence. In fact, both ranges of convergence overlap. Estimates of these ranges are made and an example shows that within this overlapping region (between distances of 300 and $2,000 \mathrm{~km}$ ) both theories give the same result. Using this fact calculations of frequency spectra are possible which in the case of a white noise dipole show some similar features to measured frequency spectra of lightning discharges.
\end{abstract}

\section{Introduction}

Electromagnetic waves at frequencies below 100 $\mathrm{kc} / \mathrm{s}$ are reflected during daytime at the bottom of the ionosphere at about 70 to $80 \mathrm{~km}$ height. At this height the collision number is still greater than the angular frequency $\omega$, and the depth of penetration of these waves into the ionosphere is limited. For distances greater than about $500 \mathrm{~km}$ and frequencies below about $50 \mathrm{kc} / \mathrm{s}$ the actual ionosphere during the day can be replaced to a good order of approximation by a homogeneous isotropic plasma with conductivity $\sigma_{i}=\frac{N_{e} e^{2}}{m \nu}$ and a sharp lower boundary. ( $N_{e}$ and $\nu$ are mean values of electron density and collision number respectively, and $\sigma_{i}$ is of the order $10^{-8} \Omega^{-1} \mathrm{~cm}^{-1}$.)

For the "medium" distances between about 500 $\mathrm{km}$ and the limiting geometric skip distance of the first hop wave $(1,700 \mathrm{~km}$ at $70 \mathrm{~km}$ reflection height) higher order terms both in mode theory and ray theory may be important. It is the purpose of this paper to compare mode theory and ray theory in this distance range.

\section{Setting up the Equations}

We wish to calculate the vector potential of the commonly assumed vertical dipole within the system: flat earth and isotropic homogeneous ionosphere with sharp lower boundary. Following the method of H. Weyl [1919] it can be shown that the vector potential of this vertical dipole is

$\Pi(\rho, \phi, z)=-\frac{j k \vec{p}}{2 \pi} \int_{0}^{\pi / 2+i \infty} \int_{0}^{2 \pi} e^{j k \pi S \cos \left(\phi^{-} \phi^{\prime}\right)} S d \theta^{\prime} d \phi^{\prime}$

\footnotetext{
${ }^{1}$ Contribution from Heinrich-Hertz Institut, Berlin-Charlottenburg, Germany
} (Western Zone)

$$
\begin{gathered}
{\left[e^{-j k C\left|z_{0}-z\right|}+R_{e} e^{-j k C\left(z_{0}+z\right)}+\sum_{n=1}^{\infty} R_{i}^{n} R_{e}^{n-1}\left\{e^{-j k C\left(2 n h-z_{0}-z\right)}\right.\right.} \\
\quad+R_{e} e^{-j k C\left(2 n h+z_{0}-z\right)}+R_{e} e^{-j k C\left(2 n h-z_{0}+z\right)} \\
\left.\left.\quad+R_{e}^{2} e^{-j k C\left(2 n h+z_{0}+z\right)}\right\}\right] 0 \leqq z \leqq h
\end{gathered}
$$

with

$$
\begin{gathered}
S=\sin \theta^{\prime} \\
C=\cos \theta^{\prime} \\
R_{i}=\frac{n_{i}^{2} C-\sqrt{n_{i}^{2}-S^{2}}}{n_{i}^{2} C+\sqrt{n_{i}^{2}-S^{2}}} \\
n_{e}^{2}=1-\frac{j \sigma_{i}}{\epsilon_{C} \omega}
\end{gathered}
$$

$\underset{e}{\sigma_{i} \text { conductivity of }\left\{\begin{array}{l}\text { ionosphere } \\ \text { earth }\end{array}\right.}$

$(\rho, \phi, z)$ cylindrical coordinates

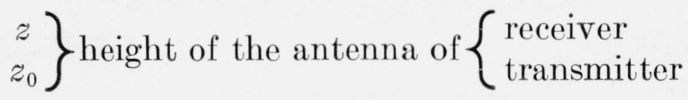

$h$ virtual height of the ionosphere

$$
\begin{gathered}
\vec{p}=\vec{p}_{0} e^{j \omega t} \text { moment of the dipole } \\
k=\frac{2 \pi}{\lambda} \text { wave number }
\end{gathered}
$$

The first two terms of the integral (1) represent the vector potential of a vertical dipole above a conductive earth without taking into account the ionosphere, a well-known problem that has been 
solved by A. Sommerfeld [1948] and which gives

$$
2 \vec{p} \frac{e^{-j k \sqrt{\rho^{2}+\left(z-z_{0}\right)^{2}}}}{\sqrt{\rho^{2}+\left(z-z_{0}\right)^{2}}} W(\alpha)
$$

$W(\alpha)=|W| e^{-j_{\Lambda}}$ is the Sommerfeld absorption factor $\alpha=-j k \rho\left[1-n_{e}\left(1+n_{e}^{2}\right)^{-1 / 2}\right]$ is the numerical distance.

Again, following Weyl [1919], the terms of the sum within the integral are approximately

$\vec{p} \sum_{n=1}^{\infty} R_{i}^{n}\left(C_{1 n}\right) R_{e}^{n-1}\left(C_{1 n}\right) \frac{e^{-j k r_{1 n}}}{r_{1 n}}+R_{i}^{n}\left(C_{2 n}\right) R_{e}^{n}\left(C_{2 n}\right) \frac{e^{-j k r_{2 n}}}{r_{2 n}}$

$+R_{i}^{n}\left(C_{3 n}\right) R_{e}^{n}\left(C_{3 n}\right) \frac{e^{-j k r_{3 n}}}{r_{3 n}}+R_{i}^{n}\left(C_{4 n}\right) R_{e}^{n+1}\left(C_{4 n}\right) \frac{e^{-j k r_{4 n}}}{r_{4 n}}$

with

$$
\begin{aligned}
r_{\gamma n}^{2} & =\left(2 n h \pm z_{o} \pm z\right)^{2}+\rho^{2} \\
C_{\gamma n} & =\frac{2 n h \pm z_{0} \pm z}{r_{\gamma n}}
\end{aligned}
$$

This (saddle-point) approximation is valid when $r_{\gamma n} \gg \lambda$ and $C_{\gamma n}$ is not too small compared with unity. $\left|n_{i}\right|$ should also be somewhat greater than unity.

Setting $z=z_{0}=0$ and neglecting higher order terms of $r_{\gamma n}$ the vertical component of the electric field strength becomes

$$
\begin{aligned}
E_{z}=2 E_{o}\left[W(\alpha)+2 \sum_{n=1}^{\infty} \sin ^{3} \theta_{n}\right. & \\
& \left.R_{i}^{n}\left(\theta_{n}\right) R_{e}^{n-1}\left(\theta_{n}\right) \frac{\left(1+R_{e}\left(\theta_{n}\right)\right)^{2}}{4} e^{j k\left(\rho-r_{n}\right)}\right]
\end{aligned}
$$

with $E_{o}=\frac{\mu_{o} \omega^{2} \vec{p}}{4 \pi} \frac{e^{-j k_{\rho}}}{\rho}$ (free space value of field strength of the vertical dipole at the distance $\rho$ )

$$
\begin{aligned}
\sin \theta_{n} & =\frac{\rho}{r_{n}} \\
r_{n}^{2} & =\rho^{2}+(2 n h)^{2}
\end{aligned}
$$

Equation (4) was derived explicitly by Wait and Murphy [1957], using a geometrical-optical approach. It can be interpreted in the language of ray-optics as consisting of the ground wave, a wave reflected from the ionosphere, and further waves reflected between the ground and the ionosphere any number of times.

Carrying out the integration over $\phi^{\prime}$ and forming the sum, (1) becomes

$$
\begin{array}{r}
\Pi(\rho, \phi, z)=\frac{j k \vec{p}}{2} \int H_{o}^{(2)}(k S \rho)\left\{e^{-j k C\left|z-z_{o}\right|}+f_{1} e^{-j k C\left(z+z_{o}\right)}\right. \\
\left.+f_{2} e^{+j k C\left(z-z_{o}\right)}\right\} S d \theta^{\prime} 0 \leqq z \leqq h
\end{array}
$$

with

$$
\begin{aligned}
& f_{1}=\frac{R_{e}\left(1+R_{i} e^{-2 j k C\left(h-z_{o}\right)}\right)}{1-R_{i} R_{e} e^{-2 j k h C}} \\
& f_{2}=\frac{R_{i}\left(R_{e} e^{-2 j k h C}+e^{-2 j k C\left(h-z_{o}\right)}\right)}{1-R_{i} R_{e} e^{-2 j k h C}}
\end{aligned}
$$

Following Budden [1951] and Alpert [1955] (who set $R_{e}=1$ ) equation (5) may be approximated by a sum of modes. For $z=z_{0}=0$, it has the form

$$
\Pi(\rho, \phi, 0)=-\frac{2 \pi \overrightarrow{j p}}{h} \sum_{n=0}^{\infty} \frac{H_{o}^{(2)}\left(k S_{n} \rho\right)}{\left[1+\frac{j}{2 k h R_{i}} \cdot \frac{d R_{i}}{d C}\right]_{C=C_{n}}}
$$

Here the values $C_{n}$ are determined from the eigenvalue equation

$$
R_{i}\left(C_{n}\right) e^{-2 j h k C_{n}}=e^{-2 j n \pi} ; n=0,1,2, \ldots .
$$

The vertical component of the electric field is then (using the approximation of the Hankel function $H_{0}^{(2)}(x)$ valid for large values of $\left.x\right)$

$$
E_{z}=2 E_{0} \frac{\sqrt{\rho \lambda}}{h} e^{-j \pi / 4} \sum_{n=0}^{\infty} \delta_{n} S_{n}^{3 / 2} e^{j k_{\rho}\left(1-S_{n}\right)} \quad(\lambda<<\rho)
$$

with

$$
\delta_{n}=\frac{1}{\left(1+\frac{j}{2 k h R_{i}} \frac{d R_{i}}{d C}\right)_{C=C_{n}}} \approx\left\{\begin{array}{l}
1(n \geqq 1) \\
0(n=0)
\end{array}\right.
$$

for $f>5 \mathrm{kc} / \mathrm{s}$ and $\frac{\sigma_{i}}{\epsilon_{0} \omega}$ is of the order of unity.

Equation (8) in the language of wave optics consists of the possible propagation modes. Actually it is the approximate continuation of the ray equation into another range of convergence. More general forms of eqs (6), (7), and (8) are given by Wait [1957a] and Wait and Spies [1960] for an imperfectly conducting and curved earth.

\section{Estimate of the Ranges of Convergence}

We wish to confine the sums in eqs (4) and (8) to the first five terms. This is reasonable provided that the sixth term is smaller than 1 percent of the first one. Thus, for $\frac{\sigma_{i}}{\epsilon_{0} \omega}=1$, we get from eq (4)

$$
2 \sin ^{3} \theta_{5}\left|R_{i}^{5}\left(\theta_{5}\right)\right|<10^{-2}
$$

or

$$
\tan \theta_{5}=\frac{\rho}{8 h}<2.7 .
$$

From eq (8) we find

$$
\left|\left(\frac{S_{6}}{S_{1}}\right)^{3 / 2} e^{j k_{\rho}\left(S_{1}-S_{6}\right)}\right|<10^{-2}
$$


with

$$
\begin{aligned}
& S_{n} \approx \bar{S}_{n}-\frac{j \sqrt{2}}{k h} \frac{\bar{C}_{n}^{2}}{\bar{S}_{n}} \\
& \bar{C}_{n}=(n-1 / 2) \frac{\pi}{k h}, \\
& \bar{S}_{n}^{2}+\bar{C}_{n}^{2}=1 .
\end{aligned}
$$

Equation (11) is the first approximation of the eigenvalue-equation (7) according to J. R. Wait [1957a].

Because we have

$$
\left|\frac{S_{6}}{S_{1}}\right| \approx 1
$$

eq (10) becomes

$$
e^{-\gamma_{6}}<10^{-2} \text { with } \gamma_{6} \approx \frac{\rho \sqrt{2}}{h} \bar{C}_{6}^{2}
$$

or

$$
\frac{\rho \lambda^{2}}{h^{3}}>0.4
$$

The range of small convergence of eq (8) simultaneously is the range of good convergence of eq (4) and vice versa. In fact, both ranges overlap.

\section{Comparison Between Ray Theory and Mode Theory}

Equations (4) and (8) give only approximate results of equation (1). To get an idea of their accuracy examples have been calculated with the following parameters:

$$
R_{e}=1 ; \frac{\sigma_{i}}{\epsilon_{0} \omega}=1 ; f=15 \mathrm{kc} / \mathrm{s} .
$$

For the determination of the eigenvalues $C_{n}$ (eq (7)) tables have been used which are reported elsewhere [Volland, 1959]. Table 1 contains values of the argument

$$
\left|\frac{E_{z}}{2 E_{0}}\right|
$$

and the phase of

$$
\frac{E_{z}}{2 E_{0}}
$$

depending on the distance $\rho$ with an ionosphere of constant height $h=70 \mathrm{~km}$, calculated from eq (4) and eq (8), respectively. Table 2 shows the same values depending on the height $h$ and with constant distance $\rho=1,000 \mathrm{~km}$. Figure 1 is a graphical form of tables 1 and 2 .

From tables 1 and 2 it can be seen that between 300 and $2,000 \mathrm{~km}$ distance and between 35 and 100 $\mathrm{km}$ height, respectively, both equations give the same result to within about 5 percent in argument. Figure 2 illustrates the influence of the higher order terms for the case $h=70 \mathrm{~km}$. In figure $2 \mathrm{a}$, corresponding to eq (4), the distance $\mathrm{O}-\mathrm{A}$ represents the constant ground wave. The vector drawn from A to one of the circles represents the first hop wave, the thin lines being equivalent to the vectors of second, third, and fourth hop waves, respectively. The vector drawn from the point $\mathrm{O}$ to the solid line which connects the end points of the thin lines gives the values of argument and phase for the different distances tabulated in table 1 , second and fourth row.

Figure $2 \mathrm{~b}$ shows the first five terms of the sum

$$
\sum_{n=1}^{\infty}\left(\frac{S_{n}}{S_{1}}\right)^{3 / 2} e^{j k \rho\left(S_{1}-S_{n}\right)}
$$

\begin{tabular}{|c|c|c|c|c|c|c|c|c|c|c|c|c|c|c|c|c|c|}
\hline$\rho$ & 0 & 100 & 200 & 300 & 400 & 500 & 600 & 700 & 800 & 900 & 1000 & 1250 & 1500 & 1750 & 2000 & 2500 & 3000 \\
\hline $\begin{array}{l}a_{w} \ldots \\
a_{r} \\
p_{w-} \\
p_{r} \ldots \ldots\end{array}$ & $\begin{array}{l}1.00 \\
0\end{array}$ & $\frac{1.04}{3}$ & $\frac{0.88}{7^{-8}}$ & $\begin{aligned} & 1.45 \\
& 1.37 \\
&-8 \\
&-3\end{aligned}$ & $\begin{array}{l}0.94 \\
32.93 \\
35\end{array}$ & $\begin{array}{r}0.24 \\
-58 \\
-54 \\
-53\end{array}$ & $\begin{aligned} & 1.31 \\
& 1.38 \\
&-51 \\
&-48\end{aligned}$ & $\begin{aligned} & 1.63 \\
& 1.54 \\
- & 39 \\
- & 33\end{aligned}$ & $\begin{aligned} & 1.97 \\
& 2.00 \\
- & 31 \\
- & 28\end{aligned}$ & $\begin{aligned} & 2.54 \\
& 2.46 \\
&-18 \\
&-13\end{aligned}$ & $\begin{array}{l}2.68 \\
2.64 \\
3 \\
7\end{array}$ & $\begin{array}{l}2.01 \\
2.05 \\
26 \\
33\end{array}$ & $\begin{array}{l}1.64 \\
1.56 \\
15 \\
22\end{array}$ & $\begin{array}{l}2.41 \\
2.34 \\
20 \\
27\end{array}$ & $\begin{array}{l}2.62 \\
2.68 \\
44 \\
51\end{array}$ & $\begin{array}{c}2.32 \\
62 \\
2\end{array}$ & $\begin{array}{r}2.74 \\
87^{-}\end{array}$ \\
\hline
\end{tabular}

in the same sequence as in figure 2a. The distance $\mathrm{O}-\mathrm{A}$ is equal to the constant first term 1, etc. The

\begin{tabular}{|c|c|c|c|c|c|c|c|c|c|c|c|c|c|c|c|}
\hline$h$ & 20 & 30 & 35 & 40 & 45 & 50 & 55 & 60 & 65 & 70 & 75 & 80 & 85 & 90 & 100 \\
\hline 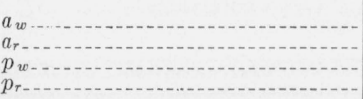 & $\begin{array}{r}0.07 \\
-455^{---} \\
-\end{array}$ & $\begin{array}{l}0.99 \\
1.30 \\
201 \\
196\end{array}$ & $\begin{array}{l}\text { 1. } 65 \\
1.78 \\
135 \\
136\end{array}$ & $\begin{array}{l}2.02 \\
2.06 \\
90 \\
84\end{array}$ & $\begin{array}{l}2.11 \\
2.07 \\
63 \\
68\end{array}$ & $\begin{array}{l}\text { 2. } 29 \\
\text { 2. } 21 \\
43 \\
47\end{array}$ & $\begin{array}{l}1.92 \\
1.95 \\
22 \\
24\end{array}$ & $\begin{array}{l}\begin{array}{l}1.67 \\
1.68 \\
27 \\
31\end{array} \\
\end{array}$ & $\begin{array}{l}2.18 \\
2.18 \\
19 \\
25\end{array}$ & $\begin{array}{l}2.68 \\
2.64 \\
3 \\
7\end{array}$ & $\begin{aligned} & 2.42 \\
& 2.39 \\
- & 23 \\
- & 20\end{aligned}$ & 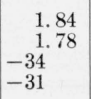 & $\begin{array}{r}1.67 \\
1.74 \\
-42 \\
-34\end{array}$ & $\begin{array}{r}1.43 \\
1.42 \\
-66 \\
-63\end{array}$ & $\begin{aligned} & 0.05 \\
- & .04 \\
- & 303 \\
- & 330\end{aligned}$ \\
\hline
\end{tabular}
vector drawn from the point $\mathrm{O}$ to the solid line must be multiplied by the first term of eq (8)

$$
\frac{\sqrt{\rho \lambda}}{h} e^{-j \pi / 4} S_{1}^{3 / 2} e^{j k \rho\left(1-S_{1}\right)}
$$

to get the values in the first and third row of table 1 .

TABLE 1. Argument $a$ and phase $p$ of $E_{z} / 2 E_{o}$ as a function of the distance $\rho$ with constant $h=70 \mathrm{~km}$ according to equation (4) (symbol " $r$ ") and equation (8) (symbol " $w$ ").

TABLE 2. Argument $a$ and phase $p$ of $E_{z} / 2 E_{0}$ as a function of the height $h$ with constant $\rho=1,000 \mathrm{~km}$ according to equation (4) (symbol "r") and equation (8) (symbol "w"). 


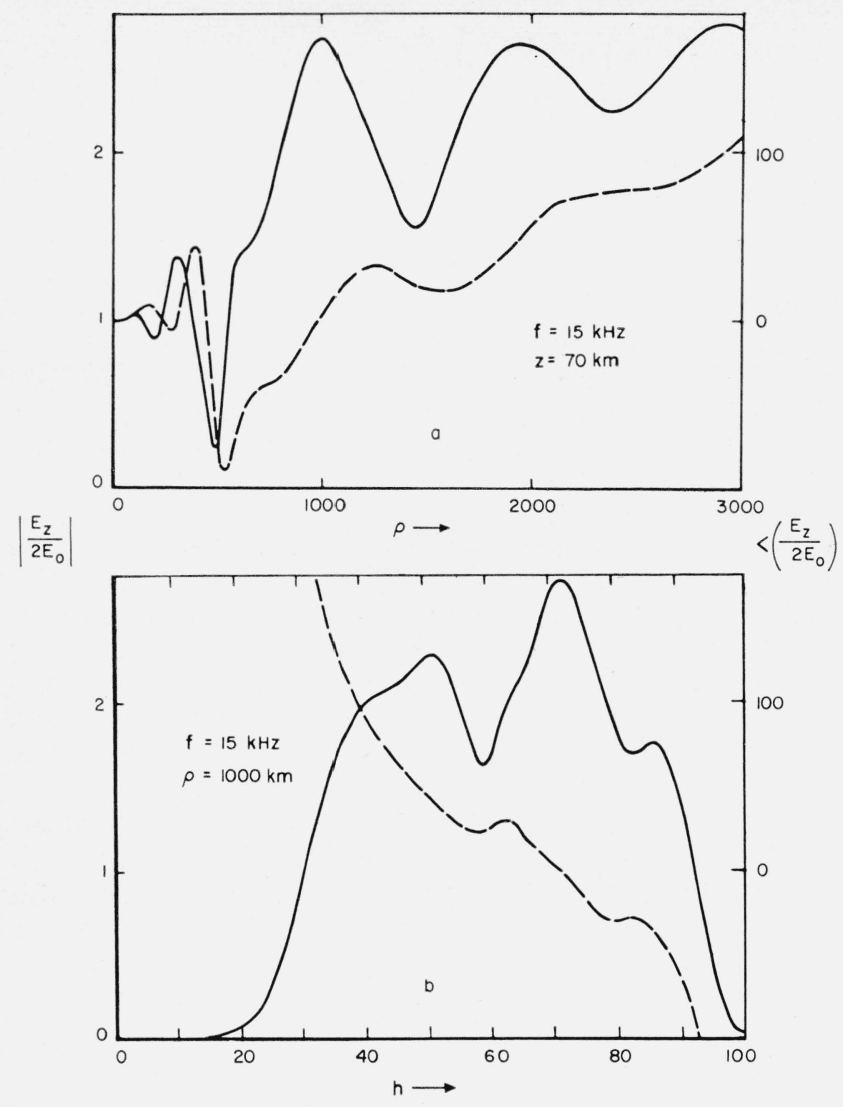

Figure 1. Argument a (solid line) and phase $p$ (dashed line) of $E_{\mathbf{z}} / 2 E_{0}$ depending on $(a)$ distance $\rho$ and $(b)$ height $h$.

From figure 2 can be seen the way in which the higher order terms of eq (4) increase and the higher order terms of eq (8) decrease with increasing distance. The minima of argument in figure 1a, beginning with the chief minimum at about $500 \mathrm{~km}$, result from the interference between $(n-1)^{\text {th }}$ and $n^{\text {th }}$ hop waves and from the interference between the first and second order mode, respectively, as can be seen from figure 2. This is in accordance with an idea from H. Poeverlein [1959].

\section{Discussion}

The strong equivalence between ray- and modetheory as shown above permits the calculation of field-strength values over a wide range of frequency, height and distance using eq (4) and eq (8) alternatively. In the case of medium distances it is also possible to replace the mode picture by the ray picture when taking into account higher order corrections of the earth's curvature and an inhomogeneous and anisotropic ionosphere. In such cases mode theory becomes very difficult, and even the calculation of the first order mode is extremely laborious [Wait and Spies, 1960]. In ray theory it is sufficient to replace the Sommerfeld ground wave by the ground wave above a curved earth according to Van der Pol and Bremmer [1949], to exchange the
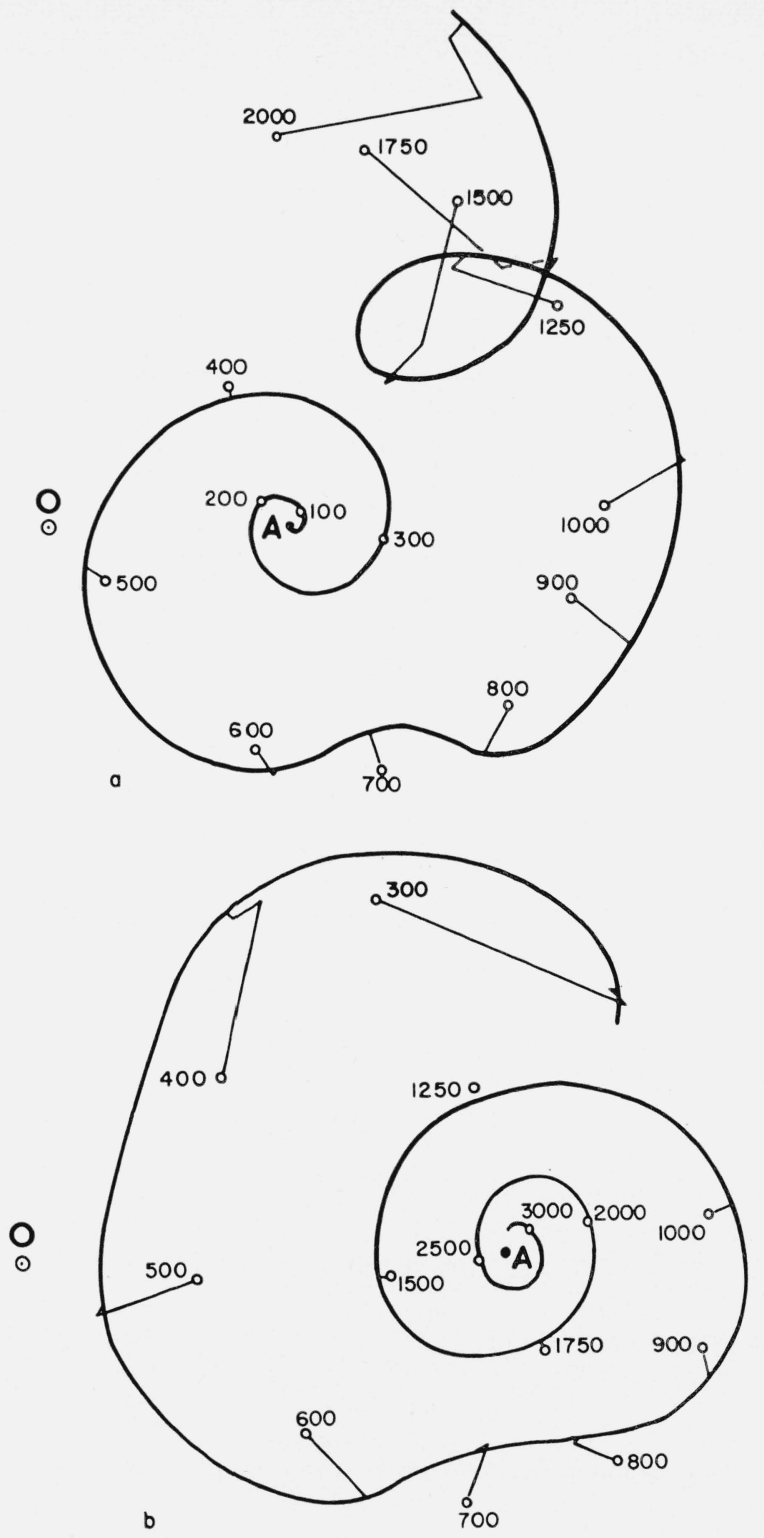

Figure 2. Sum of (a) ray-vectors and (b) mode-vectors depending on distance $\rho$ at constant height $h=\tau 0 \mathrm{~km}$.

The ratio of the scales of figure $2 \mathrm{a}$ and figure $2 \mathrm{~b}$ is $1: 2$. (For details see text.)

reflection coefficient and to correct for the earth's curvature, for the diffraction [Wait and Murphy, 1957]. Furthermore, the factor $1+R_{e}\left(\theta_{n}\right)$ must be replaced by a more complicated function which is valid when $\theta$ is near $90^{\circ}$.

Examples of calculations covering a wide range of frequencies involving the use of both eqs (4) and (8) are shown in figure 3 and figure 4 . Figure 3 gives the argument of $E_{z} / 2 E_{o}$ depending on frequency for the constant heights $h=70 \mathrm{~km}$ (solid line) and $h=60 \mathrm{~km}$ (dashed line). These curves are equivalent to the complex transfer ratio of a white noise dipole. 


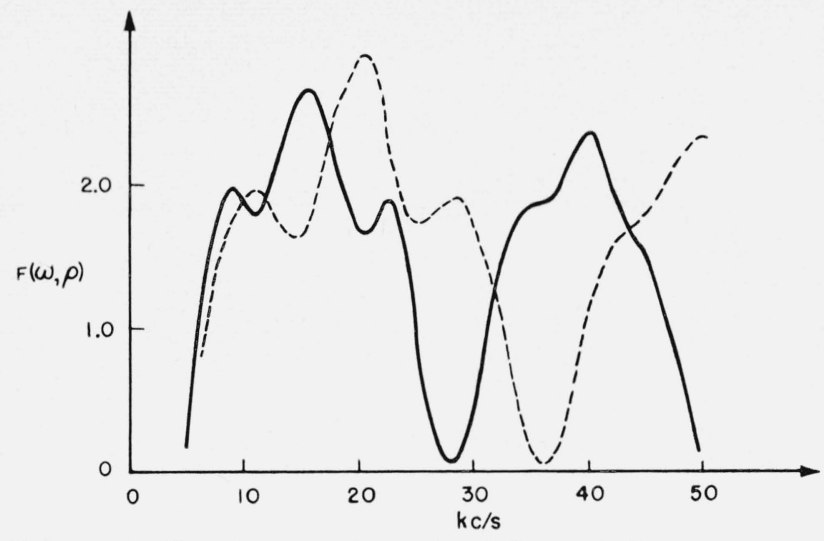

Figure 3. Complex transfer ratio of the vertical components of the electric field of a white noise dipole for a distance of 1,000 $\mathrm{km}$.

Virtual height of the ionosphere $h=70 \mathrm{~km}$ (solid line) and $h=60 \mathrm{~km}$ (dashed line).
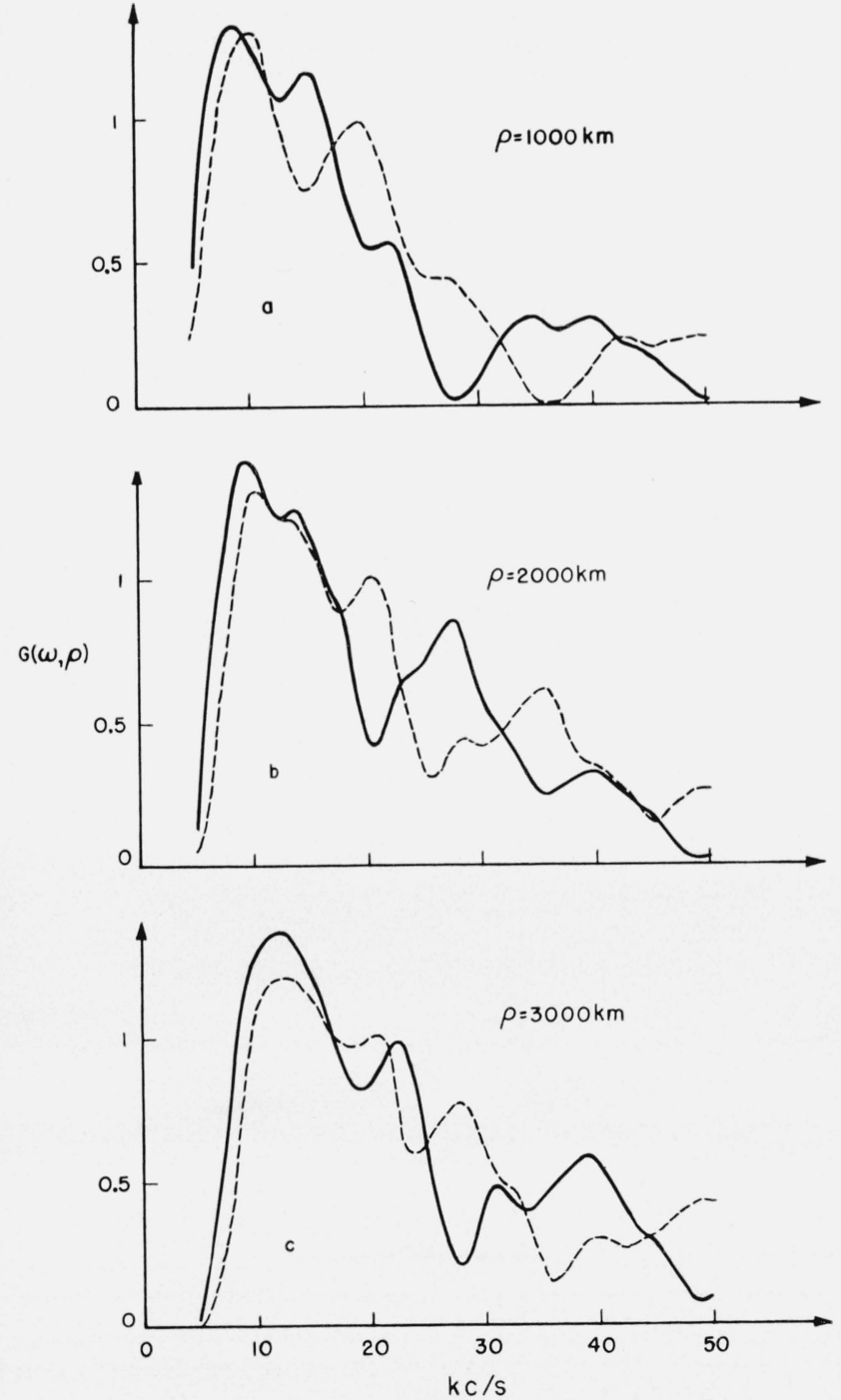

FIGURE 4. Mean frequency spectrum in the proximity of a lightning discharge multiplied by the complex transfer ratio of the vertical electric field of a white noise dipole for different distances.

(solid line: $h=70 \mathrm{~km}$; dashed line: $h=60 \mathrm{~km}$ )
These values, multiplied by the mean frequency spectrum of a lightning discharge in its proximity according to Taylor and Jean [1959], give figure 4a. They should be equal to the measured spectrum of a mean discharge at $1,000 \mathrm{~km}$ distance. Figure $4 \mathrm{~b}$ and figure $4 \mathrm{c}$ give similar calculations for distances of 2,000 and $3,000 \mathrm{~km}$, respectively. The calculated values, especially figures $4 \mathrm{a}$ and $4 \mathrm{c}$, show some similar features to the mean frequency spectra of lightning discharges measured by Obayashi et al. [1959], particularly the double maximum between 10 and $20 \mathrm{kc} / \mathrm{s}$ and the relative maximum at about $40 \mathrm{kc} / \mathrm{s}$. Very similar curves have been presented by Wait [1957b] who employed the mode sum exclusively.

During a solar flare the reflection level descends. This is represented by a $10 \mathrm{~km}$ lower level of $h=60$ $\mathrm{km}$ in figure 4 (dashed line). Measurements of field strength during such an event generally show a decrease below about $15 \mathrm{kc} / \mathrm{s}$ and an increase above $15 \mathrm{kc} / \mathrm{s}$ [Obayashi et al., 1959]. In figure 4 this is only true up to about $30 \mathrm{kc} / \mathrm{s}$. The disagreement between calculated and measured values above 30 $\mathrm{kc} / \mathrm{s}$ may be caused by neglecting second order terms of the earth's curvature, which according to Wait and Spies [1960] become more important with increasing frequency.

\section{References}

Alpert, A. L., On the propagation of VLF waves above the earth's surface, Acad. Sci. (in Russian) (USSR press, Moscow, 1955).

Budden, K. G., The propagation of radio atmospherics, Phil. Mag. 42, 833-850 (1951).

Bremmer, H., Terrestrial radiowaves (Elsevier Publishing Co., New York, N.Y., 1949)

Obayashi, T., S. Fujii, and T. Kidokoro, An experimental proof of the mode-theory of VLF ionospheric propagation, J. Geomagnetism and Geoelectricity 10, 47-55 (1959).

Poeverlein, H., Lang-und Langstwellenausbreitung, Fortschritte d. Nachrichtentechnik, Bd. 4, Frankfurt (1959).

Sommerfeld, A., Partielle Differentialgleichungen der Physik, Akad. Verlagsgellschaft Geest und Portig, Leipzig (1948).

Taylor, W. L., and A. G. Jean, Very low frequency radiation spectra of lightning discharges, J. Research NBS 63D, 199-204 (1959)

Van der Pol, B., (see Bremmer, H.).

Volland, H., Zur Theorie der Langstwellenausbreitung, Techn. Bericht d. HHI, Nr. 33, Berlin-Charlottenburg (1959).

Wait, J. R., The mode theory of VLF propagation for finite ground conductivity, Proc. IRE 45, p. 750 (1957a).

Wait, J. R., Attenuation $v s$. frequency characteristics of VLF radio waves, Proc. IRE 45, 768-777 (1957b).

Wait, J. R., Terrestrial propagation of VLF radio waves, J. Research NBS 64D, 153-203 (1960a).

Wait, J. R., Diffractive corrections to the geometrical optics of low frequency propagation, in Electromagnetic wave propagation, Academic Press, 87-101 (1960b). (Proceedings of Symposium on the Propagation of Radio Waves, Liege (1958).)

Wait, J. R., and A. Murphy, The geometrical opties of VLF sky wave propagation, Proc. IRE 45, 754-760 (1957).

Wait, J. R., and K. Spies, Influence of earth's curvature and the terrestrial magnetic field on VLF propagation, J. Geophys. Research, 65, 2325-2331 (1960).

Wevl, H., Die Ausbreitung elekromagnetischer Wellen ueber einen ebenen Leiter, Ann. Phys. 60, 481-500 (1919).

Paper 65D4-139 